\title{
Stability Analysis of Constrained Nonlinear Phase Portrait Models of Fingerprint Orientation Images
}

\author{
Jun $\mathrm{Li}^{1}$, Wei-Yun $\mathrm{Yau}^{2}$, Jiangang Wang ${ }^{2}$, and Wee Ser ${ }^{1}$ \\ ${ }^{1}$ CSP, School of EEE, Nanyang Technological University, 639798, Singapore, \\ \{junl, ewser\}@ntu.edu.sg \\ ${ }^{2}$ Institute for Infocomm Research, 21 Heng Mui Keng Terrace, 119613, Singapore \\ \{wyyau, jgwang\}@i2r.a-star.edu.sg
}

\begin{abstract}
Model based methods to compute the fingerprint orientation images were considered more robust and accurate than the gradient based methods as model based methods considered not only the local regions but also the global orientations. This paper presented the stability analysis of the constrained nonlinear phase portrait models of fingerprint orientation images. Thereafter, the constrained nonlinear phase portrait models were revised according to the stability analysis so that they were able to avoid the potential errors due to the stability. Experimental results were presented to demonstrate the revised algorithm.
\end{abstract}

Keywords: Fingerprint, Orientation Models, Stability Analysis.

\section{Introduction}

In recent decades, automatic fingerprint identification system has attracted significant interest among the scientific research community. Though large amount of effort has been made in both the academic and industrial communities, there are still unsolved problems. Noisy image processing and large scale database processing are two typical problems.

Fingerprint images consist of oriented texture. Therefore the orientation information plays a very important role in fingerprint processing. Moreover, the orientation of the fingerprint follows a certain structure that is not random. Such oriented structure provides a possibility to recover the orientation information in fingerprints corrupted by noise, even for a large noise patch which cannot be solved by the traditional gradient based methods. Accurate reconstruction of the orientations is useful for fingerprint filtering, segmentation, classification and recognition.

The orientation can be obtained using gradient based method [1] as shown in Eqn. 1].

$$
\theta\left(x_{i}, y_{i}\right)=\frac{1}{2} \arctan \left(\frac{\sum 2 G_{x} G_{y}}{\sum\left(G_{x}^{2}-G_{y}^{2}\right)}\right)+\frac{\pi}{2}
$$

where $G_{x}$ and $G_{y}$ is the gradient vectors along the $x$ and $y$ components respectively computed in the window region centered at $\left(x_{i}, y_{i}\right)$. 
This gradient based method has an assumption that there is a single dominant orientation. Otherwise, the result will not be meaningful. This assumption implies that if a fingerprint orientation has been corrupted by noise, a trustworthy orientation cannot be estimated. To overcome the noisy influence, a low pass filter is generally adopted. While a low pass filter focuses on a local region, it is not able to solve the large patch of noise.

Besides the gradient based methods, the model based methods have been proposed to improve the estimation of the orientation image [23456]. Model based methods were motivated by the fact that the fingerprint ridges flow with a certain regularity except for the limited number of singular regions. Therefore, mathematical description of such orientation field can be achieved. The most attractive feature of the orientation model is that it has capability to give a global constraint to each point in an orientation image. When reconstructing one orientation in an orientation image, all the other orientations will show their contribution. Thus, these methods are able to overcome the limitation of filterbased methods, which only considers the nearby orientations.

Several model-based methods have been proposed in the literature. we coarsely divide the model based method into two categories: Zero-Pole based method and phase portrait based methods.

\section{A: Zero-Pole Model Based Methods}

In 1993, Sherlock et al. presented an approach to model the fingerprint orientation topology [2]. The basic mathematical description of this model is given in Eqn2,

$$
O_{m}(z)=O_{0}+\frac{1}{2} \Sigma_{l=1}^{L} \arg \left(z-z_{c_{l}}\right)-\Sigma_{k=1}^{K} \arg \left(z-z_{d_{k}}\right)
$$

where $z_{c_{l}}$ and $z_{d_{k}}$ are the core point positions and delta point positions respectively; $z$ is the position of an arbitrary point; $O_{0}$ is an initial or background orientation; $O_{m}$ is the orientation at $z ; K$ and $L$ are the numbers of delta points and core points respectively.

The zero-pole model provides not a quantitative but a qualitative analysis of the fingerprint orientation as it cannot differentiate two orientation fields with the same singular points. It also cannot model the fingerprint without any singular points.

In 1996, Vizcaya et al. revised the zero-pole model to a nonlinear orientation model 3. Eqn. 3 describes this model in a mathematical form.

$$
O_{m}(z)=O_{0}+\frac{1}{2} \Sigma_{l=1}^{L} g_{c_{l}}\left(\arg \left(z-z_{c_{l}}\right)\right)-\Sigma_{k=1}^{K} g_{d_{k}}\left(\arg \left(z-z_{d_{k}}\right)\right)
$$

The difference between Eqn. 2 and Eqn. 3 is the addition of the nonlinear function $g . g$ is some family of nonlinear functions that preserve the singularity at the given point. Essentially, $g$ is decided by the mean-square orientation error between the actual orientation estimated from the fingerprint images and the orientation provided by the zero-pole model. 
Zhou and $\mathrm{Gu}$ [4 presented another complex model as shown in Eqn. 4 in 2004, which was also based on the zero-pole model while the high order rational function $f(z)$ is used as the nonlinear correction instead.

$$
O_{m}=\frac{1}{2} \arg \left[f(z) \frac{\prod_{l=1}^{L}\left(z-z_{c_{l}}\right)}{\prod_{k=1}^{K}\left(z-z_{d_{k}}\right)}\right]
$$

Zero-pole based methods have capability to recover the fingerprint orientation. While these methods encounter a problem in actual usage that they require all the singular points appearing in the image. This is not always true, especially for small sensors used for commercial purpose.

\section{B: Phase Portrait Based Methods}

Zero-pole based methods focus on obtaining the local orientation patterns near the singular point regions followed by a series of nonlinear functions to correct the global patterns. Unlike these methods, phase portrait based methods focus on obtaining the global orientation patterns first. A correction will be given to compensate the local errors near the singular points if necessary. Thus the model will not depend on the existence of the singular points.

Gu, Zhou and Zhang 5 proposed a combination model which consists of a polynomial mode 1 as in Eqn. 5 and a point charge model as in Eqn. 6. The phase portrait model is used to approximate the global orientation image and the point charge model is used to correct the orientation images formed by the phase portrait model near singular points. This combination model gives better estimation of the orientation compared to the other previous models.

$$
\begin{gathered}
\cos \left(2 \theta^{\prime}\right)=\sum_{i=0}^{n} \Sigma_{j=0}^{i} a_{(i-j) j} x^{i-j} y^{j} \\
\sin \left(2 \theta^{\prime}\right)=\sum_{i=0}^{n} \Sigma_{j=0}^{i} b_{(i-j) j} x^{i-j} y^{j} \\
P C_{c}=\left\{\begin{array}{ll}
\frac{y-y_{0}}{r} Q-i \frac{x-x_{0}}{r} Q & \mathrm{r} \leq \mathrm{R} \\
0 & \mathrm{r}>\mathrm{R}
\end{array}, P C_{d}= \begin{cases}-\frac{y-y_{0}}{r} Q-i \frac{x-x_{0}}{r} Q & \mathrm{r} \leq \mathrm{R} \\
0 & \mathrm{r}>\mathrm{R}\end{cases} \right.
\end{gathered}
$$

where $Q$ represents the strength of the singular points and $R$ denotes the radius of the region which the point charge model can affect.

The combination model empirically determines $r$ which depends on the difference between the original orientation and the phase portrait model reconstructed orientation. Furthermore, the combination model may fail where the large patch of noise appears in the image.

In 2006, Li et al. proposed a constrained nonlinear phase portrait model. Compared with $\mathrm{Gu}$ et al. approach, Li et al. used the $1^{\text {st }}$ order phase portrait model to describe the local orientation pattern near the singular points instead

${ }^{1}$ In their paper, two polynomial models are used. Here we call two polynomial models as phase portrait according to the convention in ordinary differential equations. 
of point charge model. More importantly, Li et al. used the $1^{\text {st }}$ order phase portrait as the constraint of the high order phase portrait model which was used to describe the global orientation pattern. Thus no ad-hoc parameters are necessary to combine the different models. Moreover, the $1^{\text {st }}$ order phase portrait model is able to predict the orientation when a large patch of noise appears.

In this paper, we'll give the stability analysis of the constrained nonlinear phase portrait model. According to the stability analysis, we provide the theoretical background why and how to improve the constrained nonlinear phase portrait model.

This paper is organized as follows: section 2 briefly introduces the constrained nonlinear phase portrait model, following the section 3 : stability analysis. In section 4 , experimental results are provided. Section 5 concludes the paper.

\section{Constrained Nonlinear Phase Portrait Model}

The constrained nonlinear phase portrait model is composed of two parts: (1) the $1^{\text {st }}$ order phase portrait model as given by Eqn. 7 to represent the local pattern near the singular points; (2) the high order phase portrait model to represent the global patterns as given by Eqn. 8

$$
\begin{gathered}
d x=m * x+n * y \\
d y=c * x+d * y
\end{gathered}
$$

where $\{c, d, m, n\}$ are the first order coefficients, $d x, d y$ are the x,y components of the orientation image.

$$
\begin{aligned}
\cos \left(2 \theta^{\prime}\right)= & \sum_{i=0}^{n} \Sigma_{j=0}^{i} a_{(i-j) j}\left(x-x_{s}\right)^{i-j}\left(y-y_{s}\right)^{j} \\
= & \sum_{i=0}^{n} \Sigma_{j=0}^{i} a_{(i-j) j}(-x)_{s}^{i-j}(-y)_{s}^{j}+\sum_{i=0}^{n} \Sigma_{j=0}^{i} a_{(i-j) j}(i-j)(-y)_{s}^{j} x \\
& +\sum_{i=0}^{n} \Sigma_{j=0}^{i} a_{(i-j) j} j(-x)_{s}^{i-j} y+O_{1}\left(x^{p} y^{q}\right) \quad p+q \geq 2 \\
\sin \left(2 \theta^{\prime}\right)= & \sum_{i=0}^{n} \sum_{j=0}^{i} b_{(i-j) j}\left(x-x_{s}\right)^{i-j}\left(y-y_{s}\right)^{j} \\
= & \sum_{i=0}^{n} \Sigma_{j=0}^{i} b_{(i-j) j}(-x)_{s}^{i-j}(-y)_{s}^{j}+\Sigma_{i=0}^{n} \Sigma_{j=0}^{i} b_{(i-j) j}(i-j)(-y)_{s}^{j} x \\
& +\sum_{i=0}^{n} \Sigma_{j=0}^{i} b_{(i-j) j} j(-x)_{s}^{i-j} y+O_{2}\left(x^{p} y^{q}\right) \quad p+q \geq 2
\end{aligned}
$$

where $\left\{a_{i j}\right\}$ and $\left\{b_{i j}\right\}$ are the coefficients; $O_{1}$ and $O_{2}$ are the high order items.

$$
\left\{\begin{array} { r l } 
{ \Sigma _ { i , j } a _ { ( i - j ) j } ( - x _ { s } ) ^ { i - j } ( - y _ { s } ) ^ { j } } & { = 0 } \\
{ \Sigma _ { i , j } a _ { ( i - j ) j } ( i - j ) ( - y _ { s } ) ^ { j } } & { = m } \\
{ \Sigma _ { i , j } a _ { ( i - j ) j } j ( - x _ { s } ) ^ { i - j } } & { = n }
\end{array} \quad \left\{\begin{array}{l}
\Sigma_{i, j} b_{(i-j) j}\left(-x_{s}\right)^{i-j}\left(-y_{s}\right)^{j}=0 \\
\Sigma_{i, j} b_{(i-j) j}(i-j)\left(-y_{s}\right)^{j}=c \\
\Sigma_{i, j} b_{(i-j) j} j\left(-x_{s}\right)^{i-j}
\end{array}\right.\right.
$$

where $\left\{x_{s}, y_{s}\right\}$ represent the position of a singular point.

According to the linearization theorem stated in 7]: "Let the non-linear system has a simple singular point at $\boldsymbol{x}=0$. Then in a neighborhood of the origin the phase portraits of the system and its linearization are qualitatively equivalent. 
Here a singular point at the origin of a non-linear system is said to be simple if its linearized system is simple", since the singular point in fingerprints has its own complete behavior and can be considered as the simple singular point, the nonlinear phase portrait model can be linearized at the singular point. Therefore, we have the following constraints for $\left\{a_{i j}, b_{i j}\right\}$ and $\{m, n, c, d\}$ as Eqn. 9 .

Incorporating the constraints of the $1^{\text {st }}$ order phase portrait with local descriptions of the high order phase portrait gives the constrained high order phase portrait model. Thus the proposed model incorporates the best of both the local and global descriptions, which can describe the global orientation image but still preserves an accurate local description near the singular points.

\section{Solution of Coefficients and Stability Analysis}

The solution of the coefficients $\left\{a_{i j}\right\}$ and $\left\{b_{i j}\right\}$ are obtained from the $x$ and $y$ components separately. Thus the stability analysis is also done on each component separately. To understand the stability of the solution for either $\left\{a_{i j}\right\}$ or $\left\{b_{i j}\right\}$, the following analysis takes the $\left\{a_{i j}\right\}$ as the example. The $x$ component can be represented by its corresponding coefficients $\left\{a_{(n-m) m}\right\}$ as shown in Eqn. 10 .

$$
d x\left(x_{i}, y_{j}\right)=\Sigma_{n=0}^{N} \Sigma_{m=0}^{n} a_{(n-m) m} x_{i}^{n-m} y_{j}^{m}
$$

where $N$ represents its order.

Rewrite it in a matrix form as follows:

$$
\mathrm{DX}=\mathrm{XY} \cdot \mathbf{A}
$$

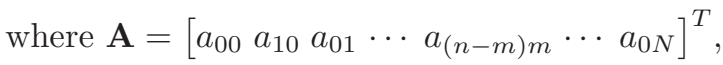

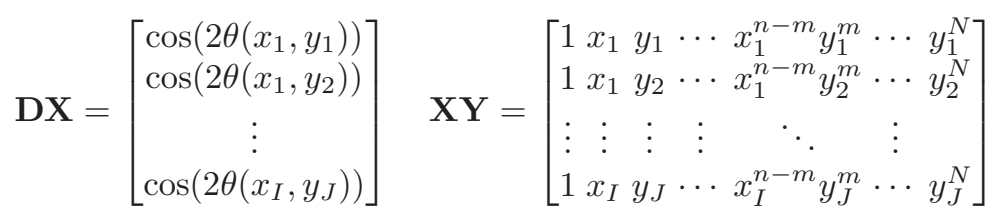

The constraints in Eqn. 9 have its matrix form as shown in Eqn. 13.

$$
\mathbf{L} \cdot \mathbf{A}=\mathbf{H}
$$

where $\mathbf{H}=[0 m n]^{T}$ and

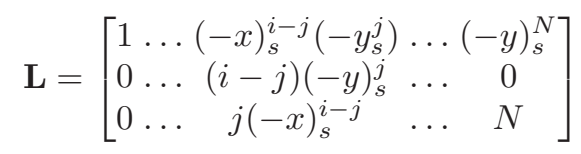

Hence the constrained nonlinear phase portrait model can be viewed as the weighted linear least square problem with linear equality constraints, as stated in the following Eqn. 15 .

$$
\min _{A \in B}\|\mathbf{W}(\mathbf{X Y} \cdot \mathbf{A}-\mathbf{D X})\|_{2}, \quad B=\{\mathbf{A} \mid \mathbf{L} \cdot \mathbf{A}=\mathbf{H}\}
$$


where $\|\cdot\|_{2}$ is the norm, $\mathbf{W}$ represents the weights which generally are represented by the corresponding coherence.

If the constrained matrix $\mathbf{L}$ has full row rank: $\operatorname{rank}(\mathbf{L})=p$, the least square problem has the unique solution, which has been proven by Eldèn [8]:

$$
\mathbf{A}=\mathbf{L}_{I \cdot W \cdot X Y^{+}} \cdot \mathbf{H}+\left(\mathbf{W} \cdot \mathbf{X Y} \cdot \mathbf{P}_{\mathbf{0}}\right)^{+} \cdot \mathbf{W} \cdot \mathbf{D X}
$$

where $(\cdot)^{+}$represents the pseudo inverse of a matrix, $\mathbf{L}_{\mathbf{I} \cdot \mathbf{W} \cdot \mathbf{X Y}}^{+}=\left(\mathbf{I}-\left(\mathbf{W} \cdot \mathbf{X Y} \cdot \mathbf{P}_{\mathbf{0}}\right)^{+}\right.$. $\mathbf{W} \cdot \mathbf{X Y}) \cdot \mathbf{L}^{+}$, and $\mathbf{P}_{\mathbf{0}}=\mathbf{I}-\mathbf{L}^{+} \cdot \mathbf{L}$.

Now let us consider the perturbation problem defined in Eqn. 17]

$$
\min _{A \in \tilde{B}}\|\tilde{\mathbf{W}} \cdot \tilde{\mathbf{X Y}} \cdot \mathbf{A}-\tilde{\mathbf{W}} \cdot \tilde{\mathbf{D X}}\|, \quad \tilde{B}=\{\mathbf{A} \mid \tilde{\mathbf{L}} \cdot \mathbf{A}=\tilde{\mathbf{H}}\}
$$

where

$$
\begin{aligned}
\tilde{\mathbf{X Y}} & =\mathbf{X Y}+\Delta X Y \mathbf{E}_{X Y} \\
\tilde{\mathbf{D X}} & =\mathbf{D X}+\Delta D X \mathbf{E}_{D X} \\
\tilde{\mathbf{L}} & =\mathbf{L}+\Delta L \mathbf{E}_{L} \\
\tilde{\mathbf{H}} & =\mathbf{H}+\Delta H \mathbf{E}_{H} \\
\tilde{\mathbf{W}} & =\mathbf{W}+\Delta W \mathbf{E}_{W}
\end{aligned}
$$

and $\left\|\mathbf{E}_{X Y}\right\|=\left\|\mathbf{E}_{D X}\right\|=\left\|\mathbf{E}_{L}\right\|=\left\|\mathbf{E}_{H}\right\|=\left\|\mathbf{E}_{W}\right\|=1$ and $\Delta X Y, \Delta L, \Delta D X$, $\Delta H, \Delta W \geq 0$.

In this study, the matrix $\mathbf{X Y}$ and $\mathbf{L}$ are combination of the coordinates of the points $(x, y)$, so we have $\Delta X Y=0$ and $\Delta L=0$.

According to the perturbation theory for the least square problem with linear equality constraints developed by Eldèn in $[\underline{8}$, the estimation errors $\Delta \mathbf{A}=\tilde{\mathbf{A}}-\mathbf{A}$ can be expressed as Eqn. 19.

$$
\begin{gathered}
\|\Delta \mathbf{A}\| \leq\left\|\left(\mathbf{X Y} \cdot \mathbf{P}_{\mathbf{0}}\right)^{+}\right\|\left\|\mathbf{W}^{+}\right\|\|\mathbf{D X}-\mathbf{X Y} \cdot \mathbf{A}\| \Delta \mathbf{W} \\
+\left\|\left(\mathbf{X Y} \cdot \mathbf{P}_{\mathbf{0}}\right)^{+}\right\| \Delta D X+\left\|\mathbf{L}_{I, X Y}\right\| \Delta H
\end{gathered}
$$

The relative error can be estimated using Eqn. 20.

$$
\begin{aligned}
\frac{\Delta \mathbf{A}}{\mathbf{A}} \leq & \frac{\Delta \mathbf{D X}}{\mathbf{D X}} \cdot \kappa_{\mathbf{L}}(\mathbf{X Y}) \frac{\|\mathbf{D X}\|}{\|\mathbf{X Y}\|\|\mathbf{A}\|}+\frac{\Delta \mathbf{H}}{\mathbf{H}} \cdot \kappa^{\mathbf{X Y}}(\mathbf{L}) \frac{\|\mathbf{H}\|}{\|\mathbf{L}\|\|\mathbf{A}\|} \\
& +\frac{\Delta \mathbf{W}}{\mathbf{W}} \kappa_{\mathbf{W}} \kappa_{\mathbf{L}}(\mathbf{X Y}) \frac{\Delta \mathbf{D X}}{\mathbf{D X}}
\end{aligned}
$$

where

$$
\begin{gathered}
\kappa_{L}(\mathbf{X Y})=\|\mathbf{X Y}\| \cdot\left\|\left(\mathbf{X Y} \cdot \mathbf{P}_{\mathbf{0}}\right)^{+}\right\| \\
\kappa^{X Y}(\mathbf{L})=\|\mathbf{L}\| \cdot\left\|\mathbf{L}_{\mathbf{I} \cdot \mathbf{X Y}}^{+}\right\| \\
\kappa_{W}=\|\mathbf{W}\|\left\|\mathbf{W}^{+}\right\|
\end{gathered}
$$

We may justify the stability of estimated coefficients $\mathbf{A}$ according to the constrained condition numbers $\kappa_{L}(\mathbf{X Y}), \kappa^{X Y}(\mathbf{L})$ and $\kappa_{W}$ defined in Eqn. 21 (a \& b \&c) and its relative error bound according to $\frac{\Delta \mathbf{A}}{\mathbf{A}}$ defined in Eqn. 20.

From Eqn. 20 the estimation error of coefficients comes from two cases: 
1. Ill conditioned matrix $\mathbf{L}$, which means that $\mathbf{L}$ tends to be singular, it will result in a large $\kappa^{X Y}(\mathbf{L})$. Therefore in this case, the estimated error of the coefficients A may be large.

2. Noise. If the perturbation of the original orientation is large, which is always indicated by the small coherence value, this will cause large $\kappa_{W}, \Delta D X$ and $\Delta W$. Therefore the estimated error of coefficients $\mathbf{A}$ will be large as well.

The solution for the $2^{\text {nd }}$ case generally required the image enhancement and noisy reduction, which is out of the scope of this paper. In this paper, we only focus on the first case.

\section{Robustness in Cases of Two Singular Points Close to One Another}

The constraints that will be added to the nonlinear phase portrait shall abide by the linearization theorem. Therefore in fingerprints the two singular points should be well separated so that each singular point exhibits a simple behavior that can be described by the first order phase portrait.

The computation of the orientation uses the pixel values in a block of size $h \times w$ (typically $8 \times 8$ or $16 \times 16$ ). Thus the orientation image has a smaller size. In some loop (or tented arch) cases, the core point and the delta point are close to each other in the orientation image. Therefore the singular point region cannot be viewed as a simple singular point. They will interact with each other such that the first order phase portrait description fail near the singular regions. Therefore the perturbation $\Delta H$ of $\mathbf{H}$ computed from the first order phase portrait is quite large. Moreover, it will cause a smaller $\left(x_{s}, y_{s}\right)$. Let us consider the constraints matrix in Eqn. 14. If $\left(x_{s}, y_{s}\right)$ is small, the elements in the first row will be smaller than the elements in the other rows. This will cause the condition number $\kappa^{X Y}(\mathbf{L})$ large, which means the $\mathbf{L}$ tends to be singular. With large $\kappa^{X Y}(\mathbf{L})$ and $\Delta H$, the estimated error of the coefficients $\mathbf{A}$ will be large. In Fig. 1 we show a fingerprint with two close singular points. Its reconstructed orientation using the $1^{\text {st }}$ order phase portrait as constraints near singular points is shown in Fig. 1 (a). It fails to precisely reconstruct the orientation at the singular point region.

In order to model the orientation in these cases, we consider the functions 22 .

$$
\begin{aligned}
& d x=x \times\left(x-x_{d}\right)+y \times\left(y-y_{d}\right) \\
& d y=-x \times\left(y-y_{d}\right)+y \times\left(x-x_{d}\right)
\end{aligned}
$$

and the orientation reconstructed using these functions is shown in Fig. 1(c).

From Fig. 1(c), we can observe the loop orientation behavior where the core and the delta are close to each other. The solutions to the Eqns. 22 are $(x=$ $0, y=0)$ and $\left(x=x_{d}, y=y_{d}\right)$. Supposing the core is at the origin and the delta is at $\left(x_{d}, y_{d}\right)$, we can coarsely reconstruct the loop orientation with constraints of two close singular points by Eqn. 22. Compared with the constraints matrix $H$, we don't use the $1^{\text {st }}$ order phase portrait to describe the singular point orientation pattern but keep the orientation of the singular points 0 . In this way, we keep the singularity of those points but no strong constraints added to cause the constraints matrix $H$ unstable. 


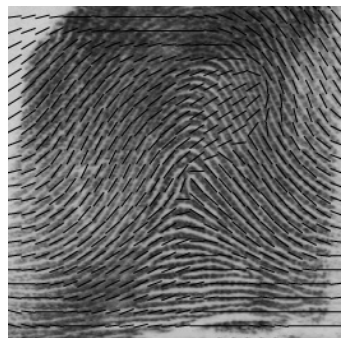

(a) by the constrained models

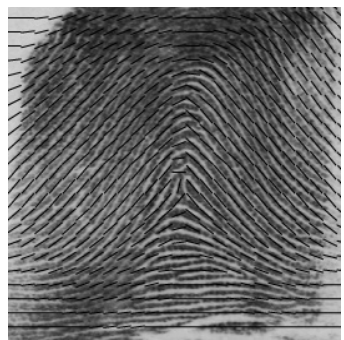

(b) by the proposed (c) model

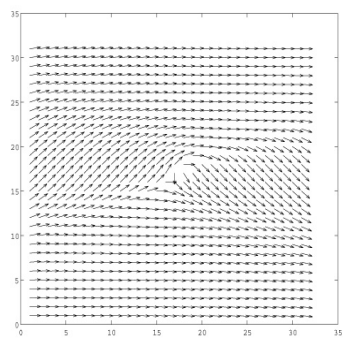

(c) Reconstructed orientation using Eqns. 22

Fig. 1. Reconstruction orientation of fingerprint with two close singular points

In order to obtain the more precise reconstructed orientation, we incorporate polynomial functions to Eqn. 22 as in Eqn. 23 .

$$
\begin{aligned}
& d x=\left(x \times\left(x-x_{d}\right)+y \times\left(y-y_{d}\right)\right) \times\left(\Sigma_{n=0}^{N} \sum_{m=0}^{n} a_{(n-m) m} x^{n-m} y^{m}\right) \\
& d y=\left(-x \times\left(y-y_{d}\right)+y \times\left(x-x_{d}\right)\right) \times\left(\sum_{n=0}^{N} \Sigma_{m=0}^{n} b_{(n-m) m} x^{n-m} y^{m}\right) \\
& \left\{\sum_{n=0}^{N} \Sigma_{m=0}^{n} b_{(n-m) m} x^{n-m} y^{m}\right\}
\end{aligned}
$$

where the items $\left\{\Sigma_{n=0}^{N} \Sigma_{m=0}^{n} a_{(n-m) m} x^{n-m} y^{m}\right\}$ and $\left\{\Sigma_{n=0}^{N} \sum_{m=0}^{n} b_{(n-m) m} x^{n-m} y^{m}\right\}$ are used to correct the overall orientation patterns other than singular point regions. In Fig. 1 (b), we show an example of the reconstructed orientation using the constraints in Eqn. 23 .

\section{Experiments}

The proposed method aims to improve the stability of constrained nonlinear phase portrait model when there are two singular points close to each other. In order to verify the accuracy of the proposed method, we select 38 images from the NIST Database 4 . The size of the image is $512 \times 480$. The block size for the orientation estimation is $16 \times 16$ pixels. In all these 38 images, there are at least two singular points close to each other (Here we set the distance between the two close singular points in 3 blocks as the close singular points according to the empirical test, which make the constrained nonlinear phase portrait model unstable). In order to reduce the effect of noise, all these 38 images have clear ridge-valley structure.

As the errors always occur at the singular point region, the difference between the original orientations and the reconstructed orientations is defined only around the singular point region (here $14 \times 14$ blocks are taken) as Eqn. 24]shows.

$$
\operatorname{Dis}\left(O_{o}, O_{M}\right)=\frac{1}{N} \sum_{O \in \Omega}\left|O_{O}-O_{M}\right|
$$

where $O_{o}$ and $O_{M}$ are the original orientation and the reconstructed orientation respectively; $\Omega$ is the area around the singular points; $N$ is the amount of the orientation in the region $\Omega$. 
Table 1. The experimental results

\begin{tabular}{lll}
\hline & Constrained model & The proposed model \\
Dis & $18.18^{\circ}$ & $9.94^{\circ}$ \\
\hline
\end{tabular}

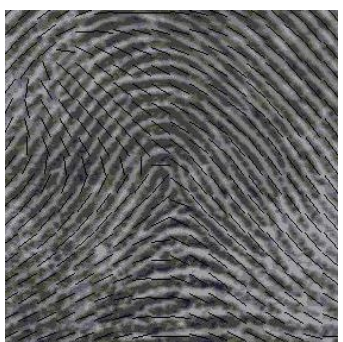

(a)

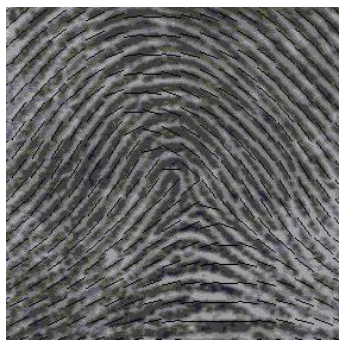

(d)

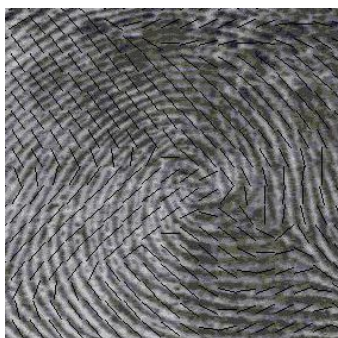

(b)

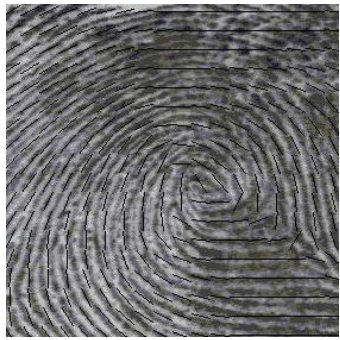

(e)

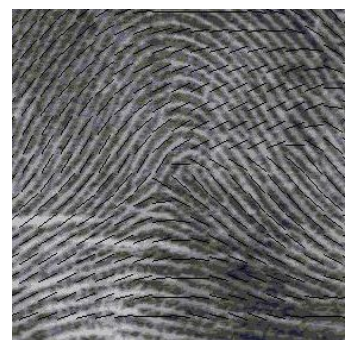

(c)

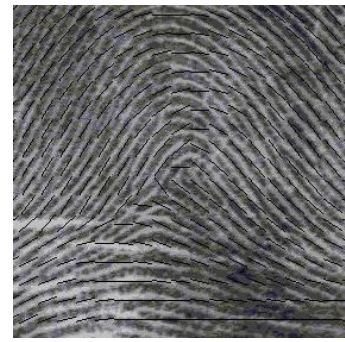

(f)

Fig. 2. Comparisons of reconstructed orientation of fingerprint with two close singular points. The first row shows the reconstructed orientation by the constrained nonlinear phase portrait model; the second row by the proposed method.

Table 1 shows the experimental results. The Dis between the original orientation and the orientation reconstructed by the constrained nonlinear phase portrait model is $18.18^{\circ}$ while the Dis between the original orientation and the orientation reconstructed by the proposed method is $9.94^{\circ}$, which has $50 \%$ improvement.

More examples are illustrated in Fig. 2. Due to the instability of the constrained matrix when the two singular points are close to each other, the reconstructed orientation becomes unpredictable. In Fig. 2 (a, b \& c), the reconstructed orientations have obvious errors. Such errors have been corrected using the proposed method as shown in Fig. 2 (d, e \& f).

\section{Conclusion}

The effectiveness of the constrained nonlinear phase portrait model has been shown by the experimental results in our early paper [6]. However, errors do occur, especially when the two singular points are close to each other. The analysis 
in this paper showed that the solution will malfunction in such case. Consequently, we propose a new model to deal with such case. The new model has only one constraint which keeps the singularity at the singular points and abandons the $1^{\text {st }}$ order phase portrait constraints as it is hard to obtain the correct $1^{\text {st }}$ order phase portrait description when the two singular points are close to each other. The experiments show that the new model has $50 \%$ accuracy improvement compared with the previous model.

\section{References}

1. Kass, M., Witkin, A.: Analyzing oriented patterns. Computer Vision Graphics and Image Processing 37(3), 362-385 (1987)

2. Sherlock, B., Monro, D.: A model for interpreting fingerprint topology. Pattern Recognition 26(7), 1047-1055 (1993)

3. Vizcaya, P., Gerhardt, L.: A nonlinear orientation model for global description of fingerprints. Pattern Recognition 29(7), 1221-1231 (1996)

4. Zhou, J., Gu, J.: Modeling orientation fields of fingerprints with rational complex functions. Pattern Recognition 37(2), 389-391 (2004)

5. Zhou, J., Gu, J., Zhang, D.: A combination model for orientation field of fingerprints. Pattern Recognition 37(3), 543-553 (2004)

6. Li, J., Yau, W., Wang, H.: Constrained nonlinear models of fingerprint orientations with prediction. Pattern Recognition 39(1), 102-114 (2006)

7. Arnold, V.I.: An Introduction to Analysis, 2nd edn. MIT Press, Cambridge (1973)

8. Eldèn, L.: Perturbation theory for the least squares problem with linear equality constraints. SIAM Journal of Numerical Analysis 17, 338-350 (1980) 